\title{
Transient effects during erosion of WN by deuterium ions studied with the quartz crystal microbalance technique
}

\author{
Bernhard M. Berger ${ }^{1 *}$, Reinhard Stadlmayr ${ }^{1}$, Gerd Meisl ${ }^{2}$, Miha Čekada ${ }^{3}$, \\ Christoph Eisenmenger-Sittner ${ }^{4}$, Thomas Schwarz-Selinger ${ }^{2}$, and Friedrich Aumayr ${ }^{{ }^{*}}$ \\ ${ }^{1}$ Institute of Applied Physics, TU Wien, Fusion@ÖAW, Wiedner Hauptstr. 8-10, 1040 Vienna, Austria \\ ${ }^{2}$ Max-Planck-Institut für Plasmaphysik, Boltzmannstraße 2, 85748 Garching, Germany \\ ${ }^{3}$ Jožef Stefan Institute, Jamova 39, 1000 Ljubljana, Slovenia \\ ${ }^{4}$ Institute of Solid State Physics, TU Wien, Wiedner Hauptstr. 8-10, 1040 Vienna, Austria
}

\begin{abstract}
Transient effects during erosion of polycrystalline tungsten-nitride (WN) films by mono-energetic deuterium projectiles are studied using a quartz crystal microbalance technique. The evolution of the mass removal rate of a $360 \mathrm{~nm}$ thin WN film under $500 \mathrm{eV} / \mathrm{D}$ and $1000 \mathrm{eV} / \mathrm{D}$ bombardment is investigated at a temperature of $465 \mathrm{~K}$ in situ and in real-time as a function of the deuterium fluence. The measurements are performed at a typical flux of $10^{18} \mathrm{~m}^{-2} \mathrm{~s}^{-1}$. A strong dependency of the observed mass change rate on the deuterium fluence is found. The mass loss is initially higher than for pure tungsten (W) and drops with fluence, finally reaching the same steady state value as for pure $W$ sputtering. Steady state surface conditions are obtained at a fluence of about $0.2 \times 10^{23} \mathrm{D} / \mathrm{m}^{2}$ for $500 \mathrm{eV} / \mathrm{D}$ and $0.6 \times 10^{23} \mathrm{D} / \mathrm{m}^{2}$ for $1000 \mathrm{eV} / \mathrm{D}$. SDTrimSP simulations indicate a preferential removal of $\mathrm{N}$ and a corresponding $\mathrm{W}$ enrichment of the surface.
\end{abstract}

Keywords: plasma wall interaction, sputtering, erosion, tungsten, tungsten-nitride 


\section{Introduction}

In magnetically confined thermonuclear fusion devices with full metal walls, impurity seeding into the plasma is required to reduce the peak power load on the divertor targets by radiative cooling [1, 2]. At ASDEX Upgrade, a Tokamak experiment with a full tungsten (W) wall [3], and JET, a Tokamak experiment with an ITER like wall [4], nitrogen impurity seeding was able to reduce the power flux to the divertor and to increase the overall plasma performance [1]. The use of nitrogen $\left(N_{2}\right)$ as a seeding gas in combination with a W divertor will lead to the formation of tungsten-nitride (WN) layers. The sputtering of $\mathrm{W}$ and $\mathrm{WN}$ by $\mathrm{N}$ is now well investigated and processes like $\mathrm{N}$ implantation, $\mathrm{N}$ retention and $\mathrm{N}$ co-deposition are well described [5-7]. In contrast to this, less is known about the interaction of $D$ plasmas with WN surfaces. In a recent study the penetration of $D$ and the removal of $\mathrm{N}$ from magnetron sputtered $\mathrm{WN}$ films was investigated under deuterium plasma exposure [8]. In this work, the erosion of thin WN films by impact of mono-energetic deuterium projectiles is studied under well-defined laboratory conditions using a highly sensitive quartz crystal microbalance technique. Results are compared to existing data for pure tungsten and SDTrimSP simulations [9-11].

\section{Experimental setup}

The measurements have been performed using a highly sensitive quartz crystal microbalance (QCM) developed at TU Wien $[9,12]$. The main part of the QCM setup is a stress compensated (SC-cut) quartz crystal driven in thickness shear mode at its resonance frequency of about $6 \mathrm{MHz}$. The total mass change of the target film under ion bombardment can be determined directly by a change of the resonance frequency according to the Sauerbrey equation [13]

$$
\frac{\Delta f}{f}=-\frac{\Delta m}{m}
$$

Both sides of the quartz crystal are covered with (140 nm thick) gold electrodes for electrical conductivity. In a Balzers Sputron triode sputter apparatus (base pressure $7 \times 10^{-6} \mathrm{mbar}$ ) at the Jožef Stefan Institute in Ljubljana a thin layer has been deposited on the top Au electrode of several quartz crystals, which consists of a $20 \mathrm{~nm}$ chromium and a $10 \mathrm{~nm}$ tungsten interlayer (for improved adhesion) followed by a $360 \mathrm{~nm}$ WN film. The composition of the WN film has been checked by Time-of-flight Elastic Recoil Detection Analysis (TOF-ERDA) using a ${ }^{127}$ l beam at $20 \mathrm{MeV}$, yielding a $\mathrm{W} / \mathrm{N}$ ratio of $1 / 1$ with approximately 5 at\% of $\mathrm{O}$ and $3 \%$ of Ar impurities [14]. A similar number of quartz crystals were prepared at TU Wien with a pure W film (thickness of typically $300 \mathrm{~nm}$ ) sputter deposited on the top gold electrode. 
A sketch of the QCM setup at TU Wien is shown in fig 1 . The quartz crystal is mounted on a sample

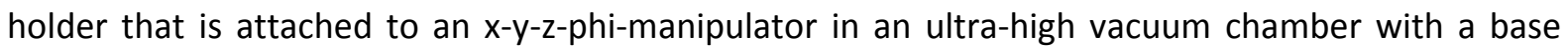
pressure in the high $10^{-9} \mathrm{mbar}$ regime. To minimize frequency drifts due to temperature changes, the whole sample holder is heated to the frequency-over-temperature minimum of the quartz crystal at typically $465 \mathrm{~K}$. A Perkin Elmer, PHI Model 04-161 Sputter Ion Gun with a Colutron Velocity Filter, Model 600 is used as an ion source with extraction voltages between $500 \mathrm{~V}$ and $2000 \mathrm{~V}$ in steps of $500 \mathrm{~V}$. To avoid neutral projectiles hitting the target film, the ion beam is bent with a pair of deflection plates. These plates and a second pair of deflection plates are also used to ensure a homogeneous irradiation of the quartz crystal by scanning the ion beam over the whole active area of the quartz crystal $\left(\geq 7 \times 7 \mathrm{~mm}^{2}\right)$ using two different zigzag voltages with frequencies of $1.6 \mathrm{kHz}$ and $50 \mathrm{~Hz}$ (for a more detailed description of the QCM technique see [9]). To ensure that the mass removal rate is not affected by a temperature change due to ion beam bombardment, the temperature of the quartz crystal microbalance holder was measured with a thermocouple and monitored during the whole measurement (for details see [15]).

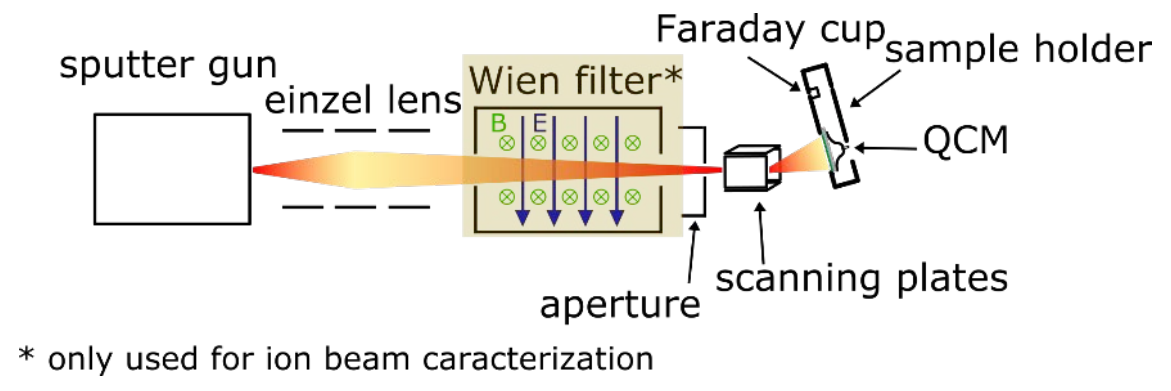

Fig. 1: Schematics of the experimental setup. For ion beam characterization the ions are produced in the Perkin Elmer ion gun and then focused and selected according to their mass (velocity) in a Wien filter. A pair of scanning plates bends and scans the ion beam across the sample holder. To increase the ion flux the Wien filter is removed after the ion beam characterization. Mass change rates are determined with a quartz crystal microbalance.

A typical deuterium flux which can be achieved with this setup is in the order of $\sim 10^{17} \mathrm{~m}^{-2} \mathrm{~s}^{-1}$ as measured with a Faraday cup located at the sample holder. The ion current density measurements are conducted before and after the exposure of the target film to the ion beam. Simulations using the SDTrimSP code $[10,11]$ indicate, that for irradiating a WN film with a $1000 \mathrm{eV} / \mathrm{D}$ ion beam a total 
fluence in the order of $10^{23} \mathrm{D} \mathrm{m}^{-2}$ is necessary in order to reach steady state conditions. Thus using the flux mentioned above, reaching steady state would take more than 10 days, which exceeds the time for which constant experimental conditions can be assumed. A removal of the velocity filter increases the flux by roughly a factor of 10 and thus significantly reduces the time needed for reaching steady state conditions. We have therefore used the setup with Wien velocity filter to first characterize the ion beam and found that the ion source mainly produces $\mathrm{D}_{2}^{+}(94.5 \%)$ ions, with rather small contributions of $\mathrm{D}^{+}(1.7 \%)$ and $\mathrm{D}_{3}{ }^{+}(2.6 \%)$. In the mass spectrum also a small amount of $\mathrm{H}_{2} \mathrm{O}^{+}(0.7 \%)$ and $\mathrm{N}_{2}^{+}(0.5 \%)$ is visible, because the velocity filter is not bakeable and cannot be pumped as well as the setup without velocity filter. By removing the Wien filter and baking the chamber we were able to reduce the amount of these impurities in the UHV system to less than 0.14 $\%$ (in detail $\mathrm{H}_{2} \mathrm{O}^{+} 0.11 \%, \mathrm{~N}_{2}{ }^{+} 0.02 \%$ and $<0.01 \%$ others), as checked by a quadrupole mass spectrometer, and to increase the ion flux to typically $10^{18} \mathrm{~m}^{-2} \mathrm{~s}^{-1}$. The systematic error introduced by treating the unfiltered ion beam as a pure $\mathrm{D}_{2}{ }^{+}$beam is estimated by using the composition of the ion beam and the kinetic energy for each projectile for the respective extraction voltage (2000 V, 1000 V). With the W sputtering yield from the book "Sputtering by Particle Bombardment 2007 by Behrisch, Eckstein" for the certain particle the expected resulting sputtering yield was calculated $S P Y=\sum S P Y_{i} \times c_{i}$ with $c_{i}$ the quantity of each projectile in $\%$ and $S P Y_{i}$ the $\mathrm{W}$ sputtering yield for each projectile in $W_{\text {atoms }} /$ atom [16]. Comparing these sputtering yield to the sputtering yield of a pure $\mathrm{D}_{2}{ }^{+}$beam results is an error below $5 \%$. In the data evaluation presented below, a $\mathrm{D}_{2}{ }^{+}$projectile will be treated as two independent $D$ atoms of the same velocity (or specific energy in eV/D atom).

\section{Experimental results}

Using freshly prepared WN films for each energy, the evolution of the mass removal rate with increasing $D$ fluence was recorded at a typical flux of $10^{18} \mathrm{~m}^{-2} \mathrm{~s}^{-1}$ for kinetic energies of $500 \mathrm{eV} / \mathrm{D}$ and $1000 \mathrm{eV} / \mathrm{D}$, respectively. Experimental results are shown in fig. 2 and compared to results of measurements performed under otherwise identical conditions on pure $\mathrm{W}$ films. For both kinetic energies, the mass loss is initially higher than for pure W. This enhancement decreases with increasing fluence, finally reaching (within the uncertainties) the steady state value of pure W erosion. For the $500 \mathrm{eV} / \mathrm{D}$ bombardment, steady state conditions are reached at a fluence of about $0.2 \times 10^{23} \mathrm{D} / \mathrm{m}^{2}$ while for the $1000 \mathrm{eV} / \mathrm{D}$ bombardment a fluence of $0.6 \times 10^{23} \mathrm{D} / \mathrm{m}^{2}$ is necessary.

For the pure $\mathrm{W}$ targets the observed mass removal rates (at both energies) are constant and do not change with the fluence. The measured steady state values of the mass removal rates are shown in fig. 3 for $W$ and $W N$ as a function of $D$ impact energy in the range of $500 \mathrm{eV} / \mathrm{D}-1000 \mathrm{eV} / \mathrm{D}$. In order 
to be able to compare our measured data (full symbols) to literature values, the mass removal rate was converted to a sputtering yield (right y axis) assuming erosion of pure tungsten. Open symbols in fig. 3 show W sputtering yields measured by Sugiyama et al. [17] with Rutherford backscattering and a total weight loss technique as well as earlier QCM results by Golczewski et al. [18]. The red curve follows a SDTrimSP simulation for pure $\mathrm{W}$ under $\mathrm{D}$ bombardment. The literature data and the SDTrimSP calculation compare well with our results.

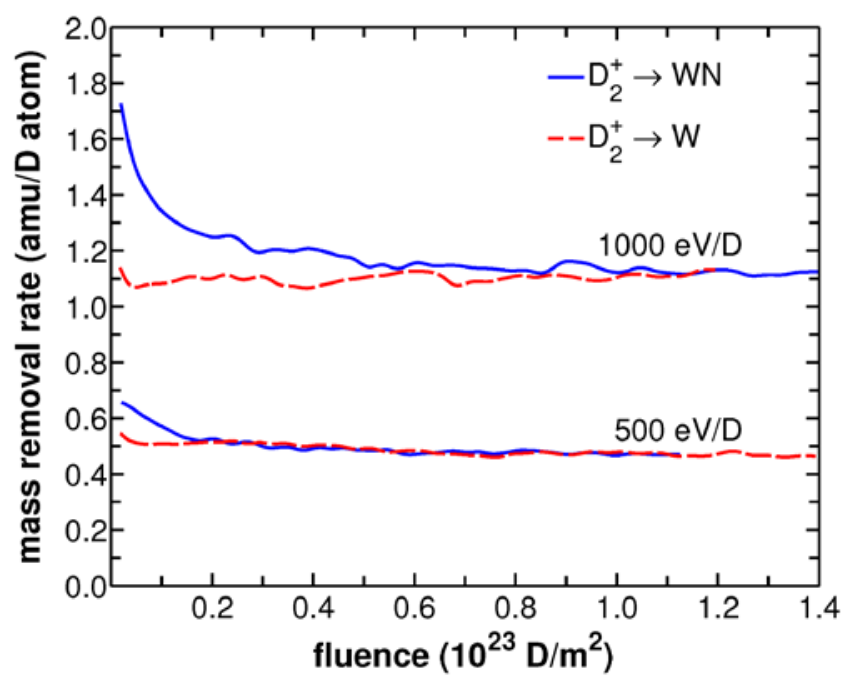

Fig. 2: Evolution of the mass removal rate of $W N$ and $W$ films as a function of the applied $D$ fluence. For both kinetic energies, a transient enhancement in the mass removal rate is seen. Steady state conditions are obtained at fluences of about $0.2 \times 10^{23} \mathrm{D} / \mathrm{m}^{2}$ and $0.6 \times 10^{23} \mathrm{D} / \mathrm{m}^{2}$ for $500 \mathrm{eV} / \mathrm{D}$ and $1000 \mathrm{eV} / \mathrm{D}$ bombardment, respectively.

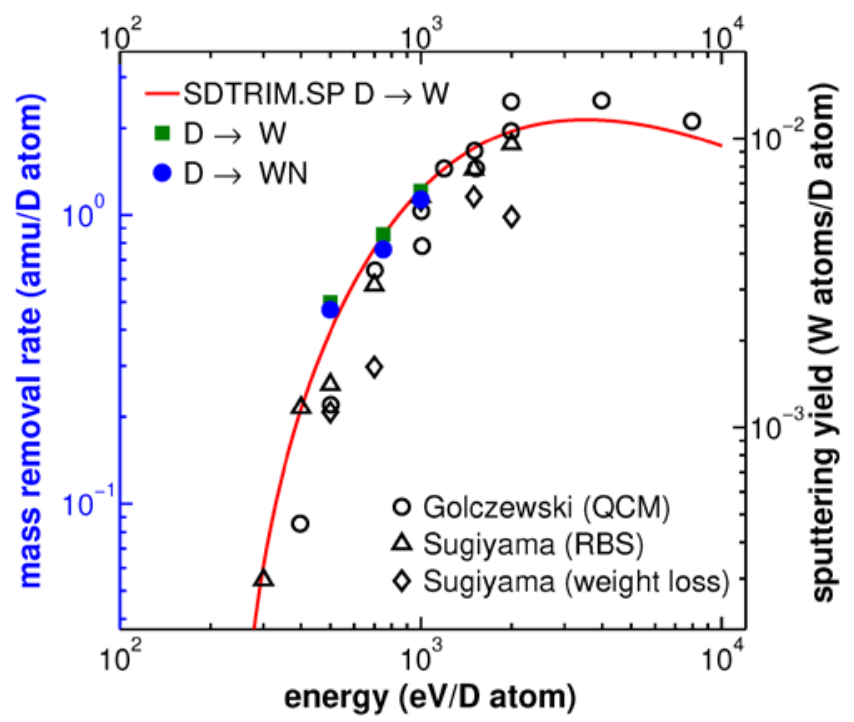


Fig. 3: Measured mass removal rates for $W$ and $W N$ (full symbols) after reaching steady state conditions as a function of the $\mathrm{D}$ energy. In order to compare the measured data to literature data on $W$ sputtering $[17,18]$ the sputtering yield (right axis) is scaled to the mass removal rate of pure tungsten. 


\section{Simulations and discussion}

To model the experimental results, the simulation code SDTrimSP $[10,11]$ has been used. SDTrimSP is a Monte Carlo code, which can calculate collision effects in (amorphous) solids like (preferential) sputtering, range profiles of implanted ions and dynamically changing composition profiles of the target using a binary-collision approximation. It should be noted that the binary collision approximation does not include thermally activated processes such as chemical erosion or diffusion.

Fig. (4) shows the evolution of the calculated $\mathrm{N}$ surface concentration with increasing $D$ fluence for a WN target with an initial composition of $50 \% \mathrm{~N}$ and $50 \% \mathrm{~W}$. The $\mathrm{N}$ and $\mathrm{W}$ surface binding energies used in the simulations change with $\mathrm{N}$ concentration based on surface binding energy values in the literature; i.e. for $N$ from $5.65 \mathrm{eV}$ (in $\mathrm{W} / \mathrm{N}=1 / 1$ ) to $6.7 \mathrm{eV}$ (in pure $\mathrm{W}$ ) $[19,20]$ and for $\mathrm{W}$ from $7.75 \mathrm{eV}$ (in $\mathrm{W} / \mathrm{N}=1 / 1$ ) to $8.8 \mathrm{eV}$ (in pure $\mathrm{W}$ ) [21]. Comparing the trend of the calculated $\mathrm{N}$ surface concentration in fig. 4 with the trend of the measured mass removal rate for WN in fig. 2, it appears likely that the decrease of the mass removal rate with fluence is caused by the decreasing $\mathrm{N}$ surface concentration. In particular, for $1000 \mathrm{eV} / \mathrm{D}$ impact the surface concentration of $\mathrm{N}$ reaches a steady state value $(10 \%-11 \%)$ at a very similar fluence $\left(0.6 \times 10^{23} \mathrm{D} / \mathrm{m}^{2}\right)$ as the corresponding mass removal rate. This can be explained as followed: During the transient initial phase a $\mathrm{N}$ depleted surface layer is produced. This dynamic state of the surface is maintained during further sputtering, because $\mathrm{N}$ is removed from the layer at the same rate as $\mathrm{W}$ is sputtered. The contribution of $\mathrm{N}$ to the mass removal rate is $\sim 7 \%$ (exactly $14 /(184+14))$. Under the circumstance that the mass removal rate for WN is reaching the same value as the mass removal rate for $\mathrm{W}$, it seems likely that the mass removal rate of WN should actually be slightly lower. But considering our experimental uncertainties, we cannot make a strong point out of this.

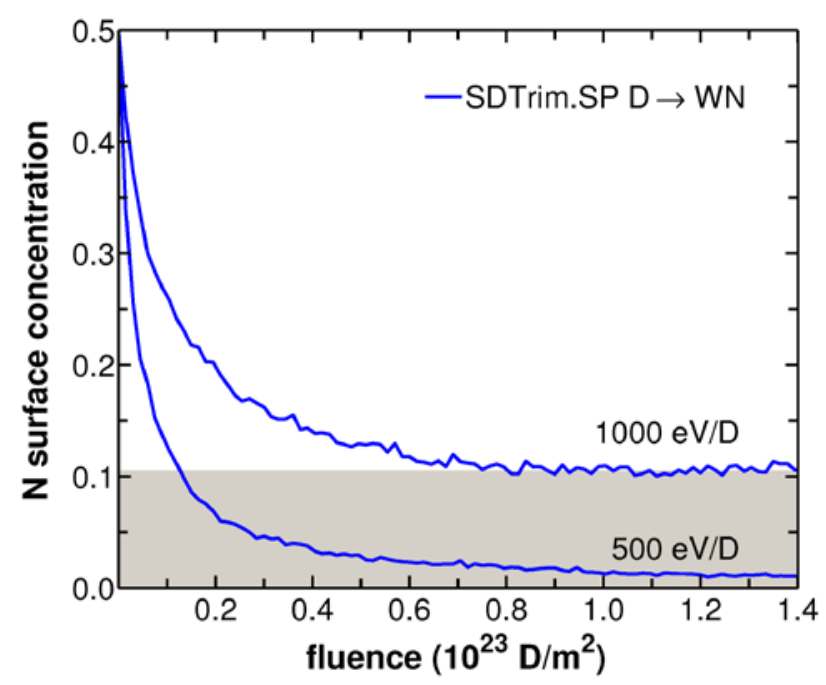


Fig. 4: $\mathrm{N}$ surface concentration as a function of $D$ fluence as calculated with SDTrimSP for a WN target with an initial composition of $50 \% \mathrm{~N}$ and $50 \% \mathrm{~W}$. For $1000 \mathrm{eV} / \mathrm{D}$ the simulated $\mathrm{N}$ surface concentration reaches a steady state value at about $10 \%$, for $500 \mathrm{eV} / \mathrm{D}$ the $\mathrm{N}$ surface concentration drops to $1 \%-2 \%$.

Despite the qualitative agreement of the simulated surface concentration and the experimentally observed fluence dependence, the mass loss rates shown in fig. 2 are not correctly reproduced by SDTrimSP. In particular the transient enhancement of the erosion yield at low incident fluences is suppressed. An adjustment of the surface binding energy values to $4.7 \mathrm{eV}$ for $\mathrm{N}$ and $6.75 \mathrm{eV}$ for $\mathrm{W}$ in the SDTrimSP simulation would fix the discrepancy, but such low values seem rather unphysical. A more likely explanation is that nitrogen diffusion, in particular irradiation-induced diffusion of nitrogen, which is not yet implemented in the SDTrimSP code, enhances the N loss. Indications that such irradiation-induced $\mathrm{N}$ diffusion occurs were already reported in [6]. In fig. 5 we compare the experimentally observed mass removal rate, with the partial tungsten mass removal rate as calculated by SDTrimSP. Assuming that the later one is approximately correctly described by SDTrimSP, the grey areas represent the amount of nitrogen that needs to be removed in order to get perfect agreement. In fig. 6 we show the nitrogen depth distribution for different $D$ fluences and a $D$ impact energy of $1000 \mathrm{eV} / \mathrm{D}$ as calculated by SDTrimSP. In the simulation the $\mathrm{N}$ depletion in the near surface region is not only caused by sputtering of $\mathrm{N}$ atoms but is also due to implantation of recoiling $\mathrm{N}$ atoms into deeper layers of the bulk, where the $\mathrm{N}$ concentration increases. In order to explain our measurements, however, an amount of nitrogen would have to be removed that corresponds to the dash-dotted depth profile in fig. 6. Considering the range distribution of $1000 \mathrm{eV} / \mathrm{D}$ projectiles, this would in principle be feasible, if (radiation-induced) diffusion of nitrogen (not accounted for in SDTrimSP) plays an important role. 


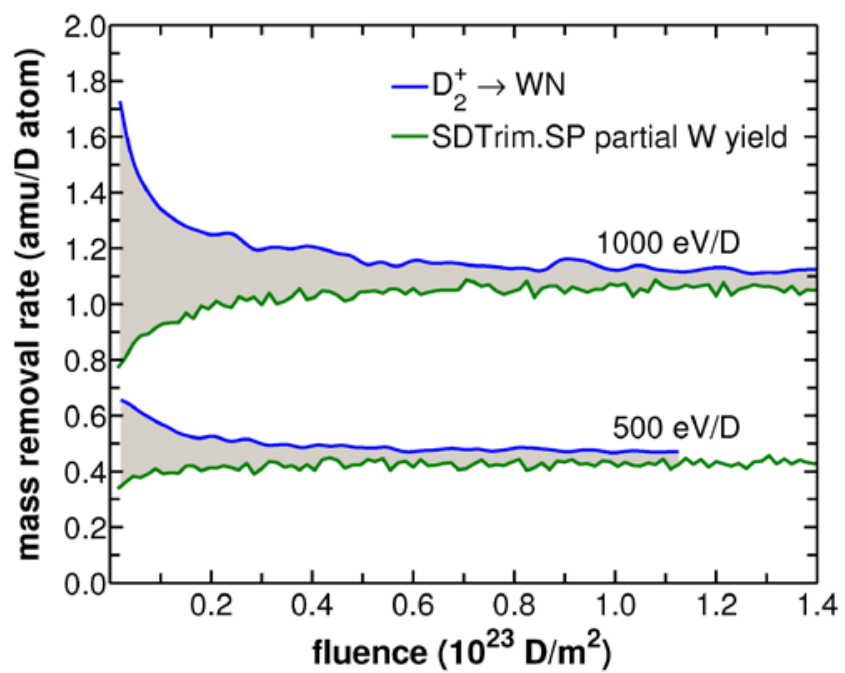

Fig. 5: Comparison of the experimentally observed mass removal rate of WN (blue) as a function of the applied D fluence with the in SDTrimSP calculated partial W mass removal rate (green) for WN. Assuming that the later one is correct, the grey area represents the amount of nitrogen that needs to be removed in order to get perfect agreement between SDTrimSP simulation and experimental results.

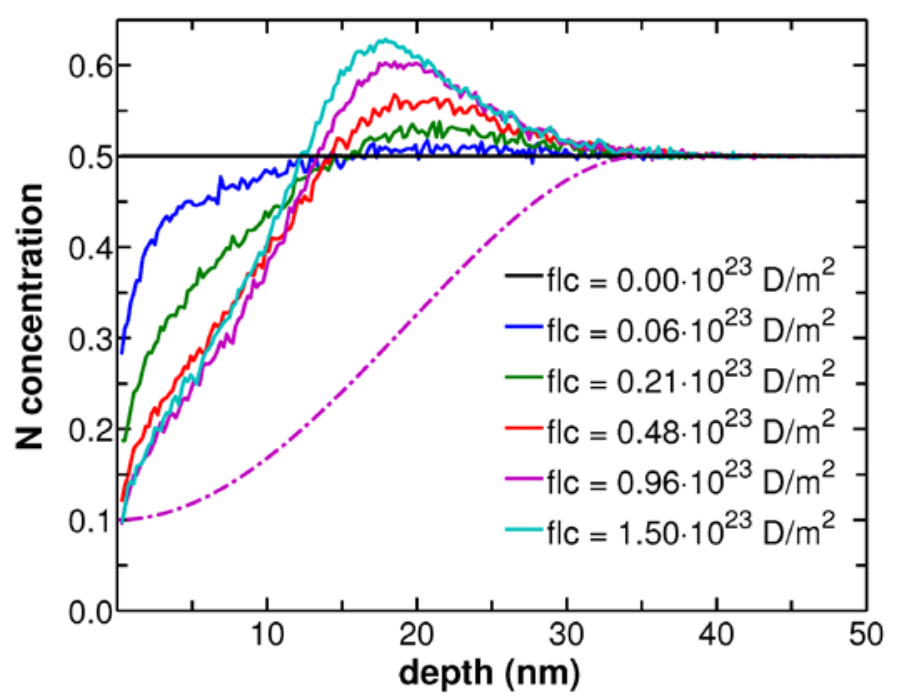

Fig. 6: Calculated $\mathrm{N}$ depth distributions for different $\mathrm{D}$ fluences (flc) and an impact energy of 1000 $\mathrm{eV} / \mathrm{D}$. The dash-dotted line shows an assumed $\mathrm{N}$ concentration profile, which would be consistent with the experimental results shown in fig. 2. 
Chemical erosion, e.g. by the formation of ammonia [22], could be another alternative explanation for the experimentally observed enhanced mass removal rate, but since $\mathrm{ND}_{3}$ will probably not be very mobile in $\mathrm{W}$, this explanation would again require the diffusion of a comparable amount of $\mathrm{N}$.

\section{Summary}

Using a QCM technique we have studied the evolution of the mass removal rate of WN films under $500 \mathrm{eV} / \mathrm{D}$ and $1000 \mathrm{eV} / \mathrm{D}$ ion bombardment and compared the results to the mass removal rate of pure $\mathrm{W}$ films and SDTrimSP simulations. For WN an enhanced mass removal rate is observed up to a fluence of $0.2 \times 10^{23} \mathrm{D} / \mathrm{m}^{2}$ for $500 \mathrm{eV} / \mathrm{D}$ and $0.6 \times 10^{23} \mathrm{D} / \mathrm{m}^{2}$ for $1000 \mathrm{eV} / \mathrm{D}$. Under steady state conditions the mass removal rate of WN approaches that of pure W. Comparing the QCM results to SDTrimSP calculations indicated, that the $\mathrm{N}$ surface concentration follows the same trend with $\mathrm{D}$ fluence as the experimentally observed mass removal rate for WN. In the simulations, however, not enough $\mathrm{N}$ is removed from the surface near regions. In order to explain our experimental results we have to invoke radiation-induced diffusion of nitrogen and/or chemical erosion (ammonia formation) to achieve good agreement between measured and simulated results.

\section{Acknowledgments}

The authors are grateful to Michael Schmid (IAP, TU Wien) for his continued support with the QCM electronics. This work has been carried out within the framework of the EUROfusion Consortium and has received funding from the Euratom research and training programme 2014-2018 under grant agreement No 633053. The views and opinions expressed herein do not necessarily reflect those of the European Commission. Financial support has also been provided by KKKÖ (commission for the coordination of fusion research in Austria at the Austrian Academy of Sciences - ÖAW).

\section{References}

[1] A. Kallenbach, M. Balden, R. Dux, T. Eich, C. Giroud, A. Huber, G.P. Maddison, M. Mayer, K. McCormick, R. Neu, T.W. Petrie, T. Pütterich, J. Rapp, M.L. Reinke, K. Schmid, J. Schweinzer, S. Wolfe, J. Nucl. Mater., 415 (2011) S19.

[2] A. Kallenbach, R. Dux, J.C. Fuchs, R. Fischer, B. Geiger, L. Giannone, A. Herrmann, T. Lunt, V. Mertens, R. McDermott, R. Neu, T. Pütterich, S. Rathgeber, V. Rohde, K. Schmid, J. Schweinzer, W. Treutterer, Plasma Phys. Control. Fusion, 52 (2010) 055002.

[3] R. Neu, et al., Plasma Phys. Control. Fusion, 49 (2007) B59. 
[4] V. Philipps, P. Mertens, G.F. Matthews, H. Maier, Fusion Eng. Des., 85 (2010) 1581.

[5] K. Dobes, P. Naderer, N. Lachaud, C. Eisenmenger-Sittner, F. Aumayr, Phys. Scripta, T145 (2011) 014017.

[6] G. Meisl, K. Schmid, O. Encke, T. Höschen, L. Gao, C. Linsmeier, New J. Phys., 16 (2014) 093018.

[7] K. Schmid, A. Manhard, C. Linsmeier, A. Wiltner, T. Schwarz-Selinger, W. Jacob, S. Mändl, Nucl. Fusion, 50 (2010) 025006.

[8] L. Gao, W. Jacob, G. Meisl, T. Schwarz-Selinger, T. Höschen, U.v. Toussaint, T. Dürbeck, Nucl. Fusion, 56 (2016) 016004.

[9] G. Hayderer, M. Schmid, P. Varga, H.P. Winter, F. Aumayr, Rev. Sci. Instrum., 70 (1999) 3696.

[10] A. Mutzke, R. Schneider, W. Eckstein, R. Dohmen, SDTrimSP Version 5.00, IPP-Report, 2011.

[11] W. Möller, W. Eckstein, J.P. Biersack, Comput. Phys. Commun., 51 (1988) 355.

[12] A. Golczewski, K. Dobes, G. Wachter, M. Schmid, F. Aumayr, Nucl. Instr. Meth. Phys. Res. B, 267 (2009) 695.

[13] G. Sauerbrey, Z. Phys., 155 (1959) 206.

[14] I.B. Radović, private communication, (2015).

[15] R. Stadlmayr, Messung von Zerstäubungsausbeuten von WN und FeW Oberflächen mit Hilfe einer Quarz-Kristall-Mikrowaage, TU Wien, 2016.

[16] R. Behrisch, W. Eckstein, Sputtering by Particle Bombardment, Springer Berlin Heidelberg2007.

[17] K. Sugiyama, private communication, manuscript in prep., (2015).

[18] A. Golczewski, A. Kuzucan, K. Schmid, J. Roth, M. Schmid, F. Aumayr, J. Nucl. Mater., 390-391 (2009) 1102.

[19] A. Allouche, J Phys Condens Matter, 28 (2015) 015001.

[20] H.F. Winters, P. Sigmund, J. Appl. Phys., 45 (1974) 4760.

[21] C.R. Company, CRC Handbook of Chemistry and Physics, CRC Press1997.

[22] M. Oberkofler, D. Alegre, F. Aumayr, S. Brezinsek, T. Dittmar, K. Dobes, D. Douai, A. Drenik, M. Köppen, U. Kruezi, C. Linsmeier, C.P. Lungu, G. Meisl, M. Mozetic, C. Porosnicu, V. Rohde, S.G. Romanelli, the ASDEX Upgrade team and JET EFDA, Fusion Eng. Des., 98-99 (2015) 1371. 